\title{
CHARACTERIZATION OF RESPIRATORY PATTERNS DURING ASSISTED MECHANICAL VENTILATION IN EXPERIMENTAL ARDS
}

\author{
Robert Huhle, Andreas Güldner, Peter M. Spieth and Marcelo Gama de Abreu \\ Pulmonary Engineering Group, Department of Anesthesiology and Intensive Care Medicine, University \\ Hospital Carl Gustav Carus, Dresden University of Technology \\ robert.huhle@tu-dresden.de
}

\begin{abstract}
Controlled mechanical ventilation with variable tidal volume, random non-correlated sequences and constant minute ventilation, was found to be advantageous especially in the therapy of acute respiratory distress syndrome (ARDS). However the properties of the tidal volume sequence might differ from the intrinsic variability of subjects respiratory control especially in anaesthetized patients with ARDS. In this contribution we characterized the variability (coefficient of variation, scaling exponent and sample entropy) of cycle-by-cycle respiratory variables in different assisted ventilation modes in a model of ARDS in pigs. While cycle-by-cycle minute ventilation varies with high inter-subject variability, tidal volume shows significant long-rang correlation during ventilation with effort proportional assist.
\end{abstract}

Keywords: respiratory signals, variability, assisted mechanical ventilation, ARDS, experimental research

\section{Introduction}

Controlled mechanical ventilation with variable tidal volume $V_{T}$ was found to be advantageous especially in the therapy of acute respiratory distress syndrome (ARDS). In the majority of experimental studies uncorrelated white noise (like) patterns derived from spontaneously breathing subjects were used to control $V_{T}$ or respiratory rate $f_{R} . f_{R}$ and $V_{T}$, respectively, were calculated using Eq. 1 for the $i^{t h}$ ventilatory cycle achieving constant $M V$ [1].

$$
M V_{i}=V_{T, i} \cdot f_{R, i}
$$

However these preliminaries pose a significant deviation from the intrinsic variability in healthy subjects. In this contribution the variability of cycle-by-cycle ventilation parameters was assessed during modes of assisted mechanical ventilation in experimental ARDS.

\section{Methods}

Experimental data: Respiratory signals used for the parameter calculation described below were obtained from a previous experimental study of our group on a surfactant depletion model of ARDS in 24 female, juvenile pigs ${ }^{1}$ [2]. During therapy each animal was randomly assigned to either pressure support (PSV), variable pressure support (vPSV) or proportional assist ventilation (PAV). While in PSV the level of pressure support was constant, it varied every cycle acc. to a normally distributed, uncorrelated pattern in vPSV with $C V=30 \%$, and was proportional to the inspiratory effort of the subject during PAV. Cycle-by-cycle $V_{T}, f_{R}$ and $M V$ are subject controlled in all three modes of ventilation by mechanical triggering the ventilator. The ventilator settings were targeted at a mean tidal volume of $V_{T}=6 \frac{m l}{k g}$.

Cycle-by-Cycle parameters: Cycle-by-cycle $V_{T}, f_{R}$ and $M V$ were derived from airflow and airway pressure signals (sampling frequency: $f_{s}=200 \mathrm{~Hz}$, measurement interval: 5 minutes ${ }^{1}$, mean $f_{R}=30 \mathrm{bpm}$ ) recorded every hour up to six hours past injury $T_{1} \ldots T_{6}$. Variability of these cycle-bycycle values was assessed quantitatively and qualitatively using the parameters detailed in Tab. 1.

Table 1: Properties of variability and parameters used to assess these properties.

\begin{tabular}{ll}
\hline Property & Parameter (Unit) \\
\hline Variability & Coefficient of variation $C V(\%)$ \\
Correlation & DFA [3] scaling exponent $\alpha()$ \\
Complexity & Sample entropy [4] $S E()$ \\
\hline
\end{tabular}

Statistical analysis: Statistical analysis was performed in R (http: / / www.r-project . org/) using two-way factorial ANOVA on general linear model (GLM, factors time and mode of ventilation). Post-hoc analysis was done using Tukeys contrasts with averaged interactions and $\mathrm{p}$ values were adjusted acc. to Holms method.

\section{Results}

Statistical analysis showed no time effects in any of the analyzed parameters. The coefficient of variation $(\mathrm{CV})$ of $V_{T}$ was significantly lower in conventional PSV compared to vPSV and PAV (Tab. 2), however the target $C V_{V_{T}}=30 \%$ was not reached in vPSV with $C V_{V_{T}}^{v P S V}=22 \%$. None of the respiration rate $f_{R}$ parameters differed significantly among modes. In minute ventilation $C V$ differed significantly between all ventilation modes with $C V_{M V}^{P A V}$ being increased/decreased compared to PSV/vPSV (Tab. 2, Fig. 1). $\alpha$-coefficients differed only in $V_{T}$ significantly, while indicating auto-correlated $\frac{1}{f}$-like noise for $f_{R}$ and $M V . \alpha_{V_{T}}$ was lower in vPSV, with values around $\alpha=0.6$, indicating

\footnotetext{
${ }^{1}$ Signals from $T_{1}$ to $T_{5}$ of length $45 \mathrm{~min}$. from 15 animals were used for DFA analysis.
} 
Table 2: Mean \pm standard deviation for each parameter averaged over $T_{1} . . T_{6}$, significant differences after ANOVA shown as PSV vs. vPSV by $\$$, for PSV vs. PAV by $\S$ and for vPSV vs. PAV by \# at $p<0.05$, respectively.

\begin{tabular}{lccccccccc}
\hline & $C V_{V_{T}}$ & $C V_{f_{R}}$ & $C V_{M V}$ & $\alpha_{V_{T}}$ & $\alpha_{f_{R}}$ & $\alpha_{M V}$ & $S E_{V_{T}}$ & $S E_{f_{R}}$ & $S E_{M V}$ \\
\hline PSV & $5.6 \pm 7$ & $10 \pm 4$ & $11 \pm 5$ & $1.16 \pm 0.2$ & $0.84 \pm 0.3$ & $0.94 \pm 0.3$ & $1.44 \pm 0.3$ & $1.44 \pm 0.5$ & $1.46 \pm 0.4$ \\
vPSV & $22 \pm 5$ & $13 \pm 6$ & $21 \pm 5$ & $0.63 \pm 0.1$ & $0.91 \pm 0.2$ & $0.78 \pm 0.2$ & $1.88 \pm 0.3$ & $1.56 \pm 0.5$ & $2.03 \pm 0.3$ \\
PAV & $16 \pm 14$ & $11 \pm 6$ & $15 \pm 9$ & $0.89 \pm 0.1$ & $0.85 \pm 0.2$ & $0.86 \pm 0.2$ & $1.43 \pm 0.7$ & $1.37 \pm 0.5$ & $1.41 \pm 0.6$ \\
\hline \multicolumn{2}{c}{$\$ \$ \#$} & $\$ \S \#$ & & & & & $\S \#$ \\
\hline
\end{tabular}

white noise with limited long-rang correlation, compared to PAV and PSV.

Complexity of variability, as assessed by Sample Entropy, showed an increased entropy of $M V$ in vPSV with values comparable to random sequences.

\section{Discussion}

The reduced variability in tidal volume, as assessed by $C V$, during PSV compared to PAV and vPSV indicates that most of the variability does not result from the variability in the inspiratory timing, but likely from the inspiratory effort (muscle and/or ventilator), assuming subject-ventilator synchrony. A similar behaviour was observed in minute ventilation. This analysis shows that minute ventilation is not constant from cycle-to-cycle in the anaesthetized state.

During PSV, the variability of $M V$ is determined mainly by variability in $f_{R}$ compared to vPSV and PAV where it is mainly determined by $V_{T}$. All $C V$ values for the PAV group displayed a high scatter, indicating a high intersubject variability of this parameter and possibly a better subject-ventilator interaction in terms of cycle-by-cycle variability. However the study results show no difference in terms of gas exchange / lung mechanics but suggest increased inspiratory effort during PAV compared to (v)PSV [2]. The main limitation of this analysis of coefficients of variance is the assumption of normal distribution in all three analysed cycle-by-cycle metrics. At least the distribution of $M V$ will not hold this assumption, as it is, depending on the covariance of $V_{T}$ and $f_{R}$, a multiplied normal distribution with an essentially different characteristic equation (Eq. 1).

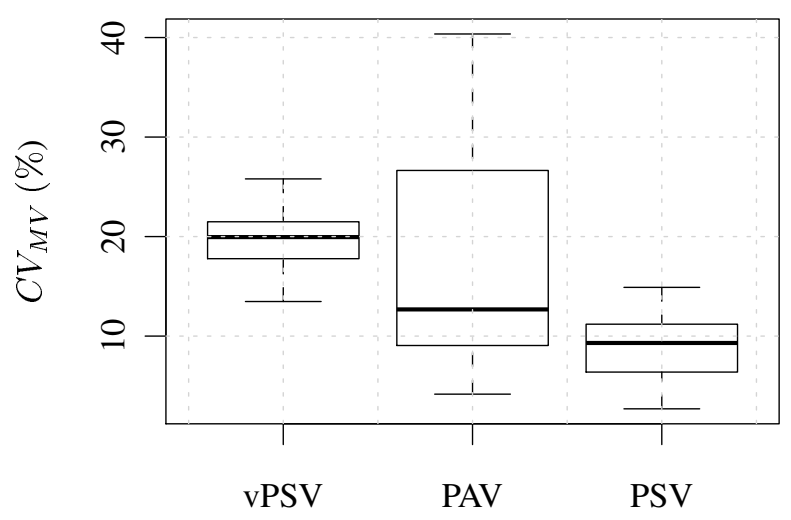

Figure 1: Dependence of $C V_{M V}$ on mode of support ventilation.
Analysis of pattern complexity showed an increased sample entropy in all parameters for vPSV similar to the value expected for a random sequence [4]. Reduced complexity in PAV suggests that subject initiated complexity is significantly lower than the complexity of a random sequence $S E_{\text {random }}>2$.

The linear detrended fluctuation analysis showed increased long-range correlation in $V_{T}$ values for PAV and PSV compared to vPSV. While the variability of PSV and its autocorrelated property mainly arise due to errors in application / measurement of $V_{T}$ and the ventilators trigger mechanism, the correlation seen for PAV can be rather attributed to the variability caused by the subject. This suggests similar long-range correlations during ARDS therapy in anaesthetized pigs and awake or REM-sleeping humans [5].

\section{Bibliography}

[1] A. Thammanomai, H. Hamakawa, E. Bartolák-Suki, and B. Suki, "Combined effects of ventilation mode and positive end-expiratory pressure on mechanics, gas exchange and the epithelium in mice with acute lung injury," PLoS ONE, vol. 8, no. 1, p. e53934, 2013. PMID: 23326543.

[2] P. M. Spieth, A. Güldner, A. Beda, N. Carvalho, T. Nowack, A. Krause, I. Rentzsch, S. Suchantke, S. C. Thal, K. Engelhard, M. Kasper, T. Koch, P. Pelosi, and M. G. de Abreu, "Comparative effects of proportional assist and variable pressure support ventilation on lung function and damage in experimental lung injury," Crit. Care Med., vol. 40, pp. 2654-2661, Sept. 2012. PMID: 22743778.

[3] J. W. Kantelhardt, E. Koscielny-Bunde, H. H. A. Rego, S. Havlin, and A. Bunde, "Detecting long-range correlations with detrended fluctuation analysis," Feb. 2001. Physica A 295, 441-454 (2001).

[4] J. S. Richman and J. R. Moorman, "Physiological timeseries analysis using approximate entropy and sample entropy," Am J Physiol Heart Circ Physiol, vol. 278, pp. H2039-H2049, Jan. 2000.

[5] S. Rostig, J. W. Kantelhardt, T. Penzel, W. Cassel, J. H. Peter, C. Vogelmeier, H. F. Becker, and A. Jerrentrup, "Nonrandom variability of respiration during sleep in healthy humans," Sleep, vol. 28, pp. 411-417, Apr. 2005. PMID: 16171285. 